\title{
Application of explicit FE codes to simulation of sheet and bulk metal forming processes
}

\author{
J. Rojek ${ }^{\mathrm{a}, *}$, E. Oñate ${ }^{\mathrm{b}}$, E. Postek ${ }^{\mathrm{a}}$ \\ a Institute of Fundamental Technological Research, Polish Academy of Sciences, ul. Swietokrzyska 21 PL-00049 Warsaw, Poland \\ ${ }^{\mathrm{b}}$ International Center for Numerical Methods in Engineering, Barcelona, Spain
}

\begin{abstract}
This paper presents the application of an explicit dynamic finite element code for simulation of metal forming processes, of both sheet and bulk forming. The experiences reported here have been gained during the development and use of our own explicit program Stampack. An original formulation of a triangular shell element without rotational degrees of freedom is reviewed combining the explicit sheet forming simulation with the implicit springback analysis as well as the parallelization of the explicit program described. An extension of a finite element code for coupled thermomechanical analysis is discussed. A new thermomechanical constitutive model developed by the authors and implemented in the program is presented. Numerical examples illustrate some of the possibilities of the finite element code developed. (C) 1998 Elsevier Science S.A. All rights reserved.
\end{abstract}

Keywords: Metal forming; Sheet stamping; Finite element simulation; Explicit dynamic analysis; Thermomechanical analysis

\section{Introduction}

Finite element programs based on the explicit dynamic formulation have proved to be a very attractive tool for the simulation of metal forming processes. Explicit time integration schemes deal with the system of discretized equations of motion in the following form:

$\mathrm{M} \ddot{\boldsymbol{r}}+\mathrm{D} \ddot{\boldsymbol{r}}=\boldsymbol{f}-\boldsymbol{p}$

where $\mathbf{M}$ and $\mathbf{D}$ are the mass and damping matrices, $\boldsymbol{r}$ is the nodal displacement vector, and $\boldsymbol{f}$ and $\boldsymbol{p}$ are the vectors of external and internal nodal forces, respectively. Employing Eq. (1) for the known configuration at time $t_{n}$ the solution for the next time instant $t_{n+1}=$ $t_{n}+\Delta t$ is obtained. The effectiveness of the explicit formulation is based on the use of a diagonal mass matrix. There are also disadvantages, the main one is the limitation of the time step due to the conditional stability. The numerical efficiency, however, and other advantages of explicit programs such as low memory requirements and easy treatment of contact conditions, cause this approach to dominate over implicit methods in industrial applications.

\footnotetext{
* Corresponding author.
}

The computation times, however, in case of large industrial problems are still quite long. The implementation of a new triangular shell element as well as the use of techniques of parallel computation reduced simulation times considerably.

The explicit program for the simulation of forming has been combined with an implicit solver to perform the analysis of elastic springback efficiently.

The use of explicit finite element programs in the analysis of sheet metal forming has become quite common, while the possibilities of this approach in the simulation of bulk forming seem to be not exploited sufficiently as yet. These problems require taking account of the thermal effects in the deformation process that can be achieved by the coupled thermo-mechanical analysis.

\section{Simple and efficient finite element formulation for sheet stamping simulation}

Efficiency is a very important factor in industrial applications of finite element simulation. It depends considerably on the finite element formulation used. Efficient solutions can be usually achieved by using elements based on simple theoretical assumptions. 
Simplicity of the formulation must not be obtained, however, at the cost of loss of its precision. The simplicity of the element developed for the simulation of sheet stamping and implemented in our code is based on avoiding the use of rotational degrees of freedom which yields a triangular shell element with three degrees of freedom per node only.

The work on the element with translational degrees of freedom only, the so-called BST (Basic Shell Triangle) element has been started by Onate and Cervera [1] and continued by Oñate and Zarate [2]. The element formulation for an explicit dynamic program has been presented in $[3,4]$.

The shell deformation is described in terms of the deformation of its midsurface. With the assumption of the Kirchhoff hypothesis the rate of deformation tensor at any point of the shell $\dot{e}=\left\{\dot{e}_{x}, \dot{e}_{x}, \dot{e}_{x}\right\}^{T}$ can be written in the following way:

$\dot{e}=\dot{\varepsilon}+z \dot{\boldsymbol{\kappa}}$

where $z$ is the distance of the current material point to the midsurface, $\quad \dot{\boldsymbol{e}}=\left\{\dot{\varepsilon}_{x x}, \dot{\varepsilon}_{y y}, \dot{\varepsilon}_{x y}\right\}^{T}$ and $\dot{\boldsymbol{\kappa}}=$ $\left\{\dot{\kappa}_{x x}, \dot{\kappa}_{y y,} \dot{\kappa}_{x y}\right\}^{T}$ are the rates of membrane and bending deformation of the midsurface, respectively.

\section{The BST element employs linear shape functions} the membrane component of the element is equivalen to the standard constant strain riangle (CST) element.
The bending part is formulated using a finite-volumelike procedure presented in [1] which allows expression of the rate of bending deformation $\dot{\kappa}$ within an element in terms of the gradient of deflection velocity $w$ along the element sides:

Register for free at https//www.scipedia.com

$\dot{\kappa}=\frac{1}{A} \int_{\Gamma} Q \nabla w \mathrm{~d} \Gamma$

where

$\boldsymbol{Q}=\left[\begin{array}{cc}n_{x} & 0 \\ 0 & n_{y} \\ n_{y} & n_{x}\end{array}\right] \quad \nabla=\left\{\frac{\partial}{\partial x}, \frac{\partial}{\partial y}\right\}^{T}$

with $A$ being an area of the element, $\Gamma$ its contour, $x$ and $y$ are the local Cartesian coordinates, and $\boldsymbol{n}=\left\{n_{x}\right.$, $\left.n_{y}\right\}^{\mathrm{T}}$ is a unit vector normal to the boundary defined in the plane of the element (Fig. 1). The deflection gradient along the element sides is in turn calculated in terms of the nodal velocities of the adjacent elements shown in Fig. 1.

The elasto-plastic model adopted for the BST element assumes a hypoelastic rate constitutive law

$\stackrel{\nabla}{\boldsymbol{\sigma}}=\boldsymbol{C}^{\mathrm{ep}} \boldsymbol{d}$

where $\underset{\nabla}{\sigma}$ is the Jaumann rate of the Cauchy stress tensor and $C^{\text {ep }}$ is the elasto-plastic constitutive tensor. Yielding is considered using the anisotropic Hill (1979) yield criterion [5].

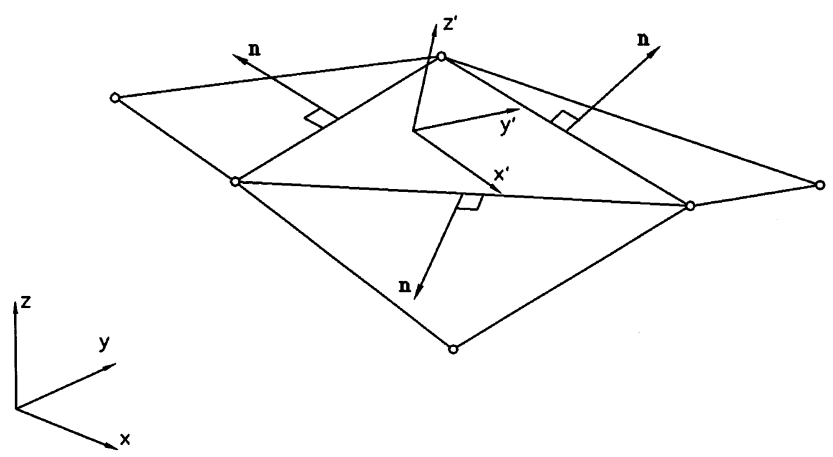

Fig. 1. Finite element patch.

The BST element has been successfully applied to sheet forming analysis with an explicit dynamic program, [6]. A number of large industrial problems have been analysed yielding good results.

\section{Springback simulation}

The dynamic analysis of forming can be extended to the springback phase-once the final deformation is

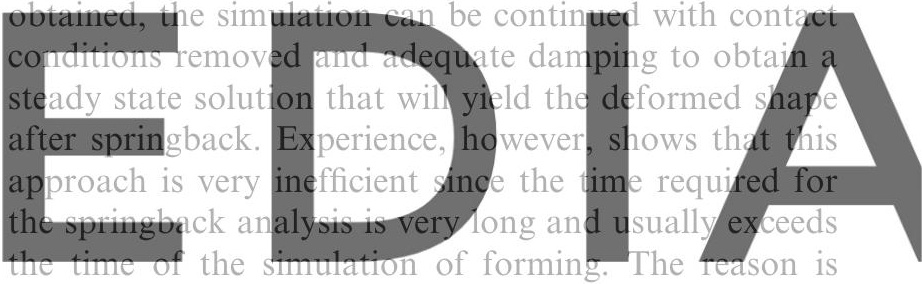

that the critical time step is very small compared with

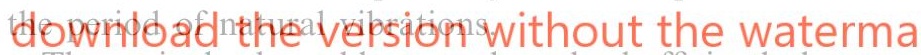
The springback problem can be solved efficiently by combining the explicit simulation of forming with implicit analysis of the springback. Both implicit dynamic and quasistatic schemes are possible. The implicit dynamic solution schemes are obtained by taking equations of motion (Eq. (1)) for the unknown configuration at time $t_{n+1}$ denoted by the subscript $n+1$ to get the solution for this configuration

$\mathbf{M} \ddot{\boldsymbol{r}}_{n+1} \mathrm{D} \dot{\boldsymbol{r}}_{n+1}=\boldsymbol{f}_{n+1}-\boldsymbol{p}_{n+1}$

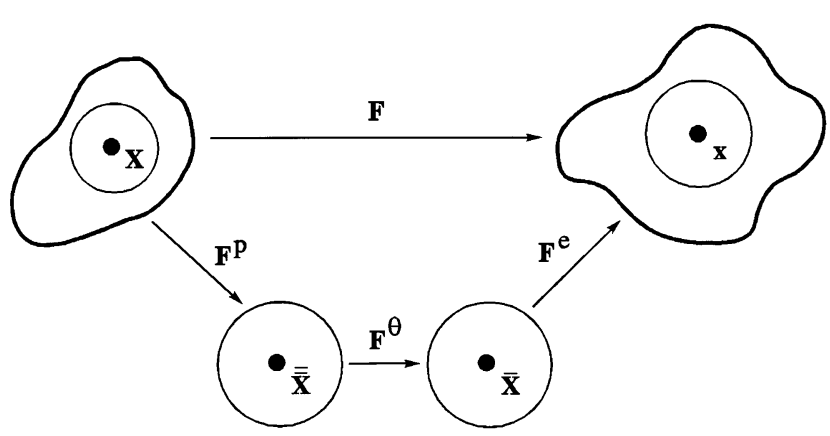

Fig. 2. Multiplicative decomposition of the deformation gradient tensor. 


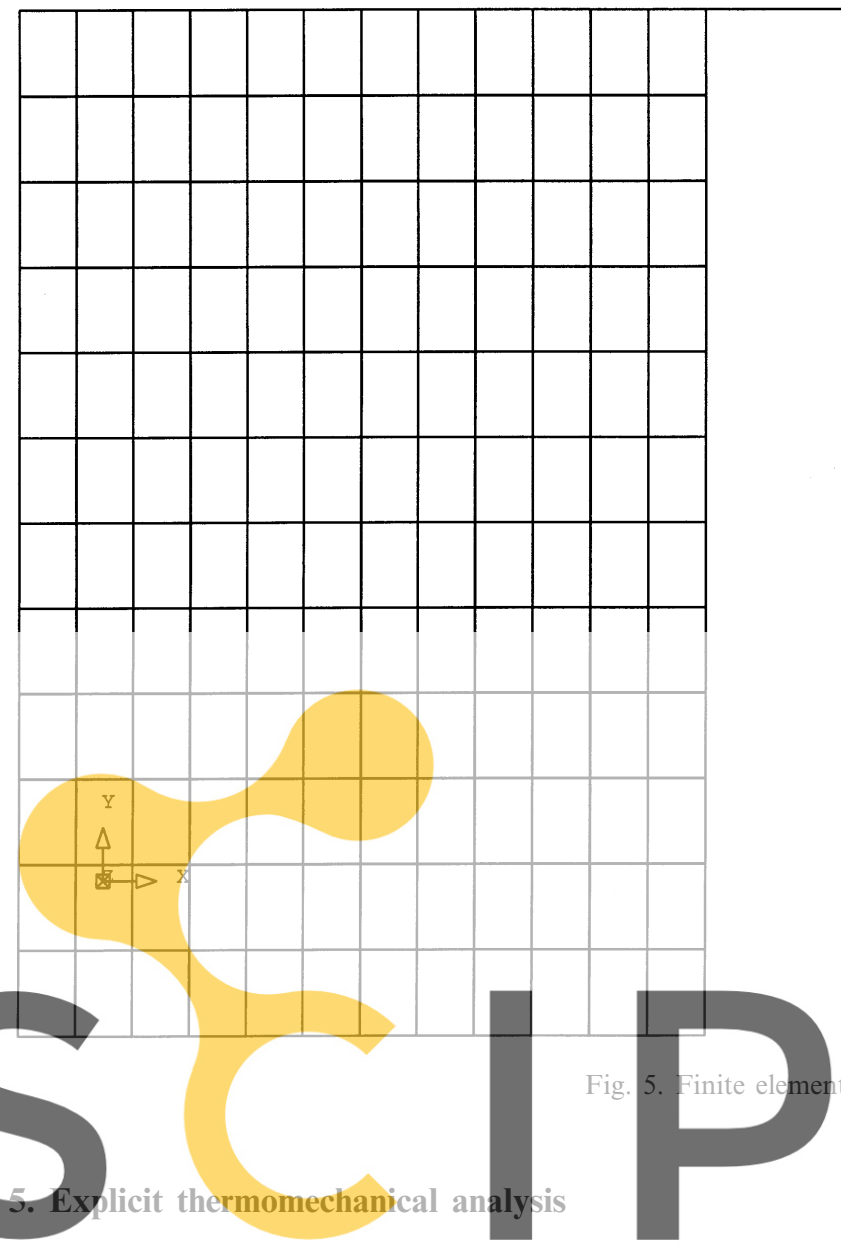

Simulation of bulk forming processes requires taking

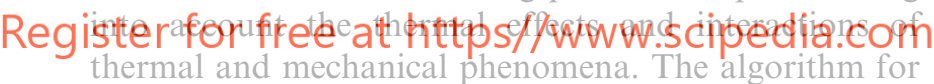
a coupled thermomechanical analysis has been implemented in our explicit program making it possible to analyse such forming processes.

In the solution of a thermomechanical problem the solution of the mechanical problem (Eq. (1)) is coupled with the solution of the heat conduction problem governed by the following discretized equation:

$\mathbf{C} \dot{\boldsymbol{T}}+\mathbf{K} \boldsymbol{T}=Q$

where $\mathbf{C}$ is the heat capacity matrix, $\mathbf{K}$ is the heat conductivity matrix, $\boldsymbol{Q}$ is the heat flux and sources vector, and $\boldsymbol{T}$ is the vector of nodal temperatures. Eqs. (1) and (9) are solved under adequate boundary conditions. Eq. (9) can be solved using either an explicit or implicit scheme of time integration. At present the forward Euler explicit time integration method has been implemented for the solution of Eq. (9) in the numerical algorithm for the thermomechanical analysis. This combined with the central difference time integration of Eq. (1) gives the following fully explicit scheme for a coupled problem:

$\ddot{\boldsymbol{r}}_{n}=\mathbf{M}_{\mathrm{D}}^{-1}\left(\boldsymbol{f}_{n}-\boldsymbol{p}_{n}-\mathbf{D} \dot{\boldsymbol{r}}_{n}\right), \quad \mathbf{M}_{\mathrm{D}}=\operatorname{diag} \mathbf{M}$
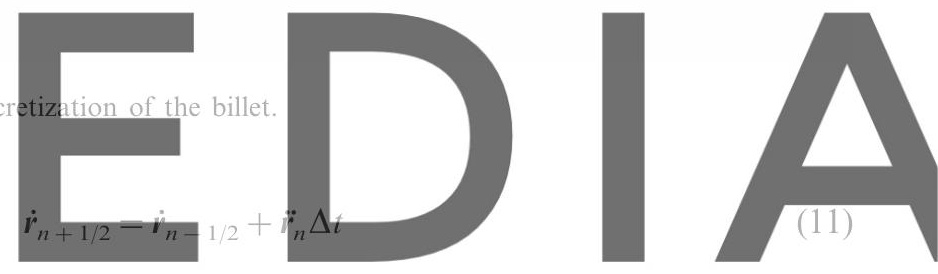

$\boldsymbol{r}_{n+1}=\boldsymbol{r}_{n}+\dot{\boldsymbol{r}}_{n+1 / 2} \Delta t$

(12)

The new configuration $r_{n+1}$ is obtained from the explicit equations of motion with the temperatures assumed fixed, and the new temperature $\boldsymbol{T}_{n+1}$ is calculated at constant geometry. The results are exchanged at each step and coupling term are calculated. Eqs. (1) and (9) for the thermo-mechanical problem are coupled by considering the following effects:

- heat generation by the plastic dissipation,

- contribution of the thermal expansion to the total material deformation,

- influence of the temperature on the yield stress of the material.

The rate of heat generation $q$ due to the plastic dissipation (contributing to the vector $\boldsymbol{Q}$ ) can be calculated as:

$q=\chi \boldsymbol{\sigma}: \boldsymbol{d}^{\mathrm{p}}$

where: $\boldsymbol{\sigma}$ is the Cauchy stress tensor, $\boldsymbol{d}^{\mathrm{p}}$ is the rate of plastic deformation tensor, and $\chi$ is the fraction of plastic work converted to heat. The method of accounting for the thermal effects in the constitutive model developed is presented in the next section. 


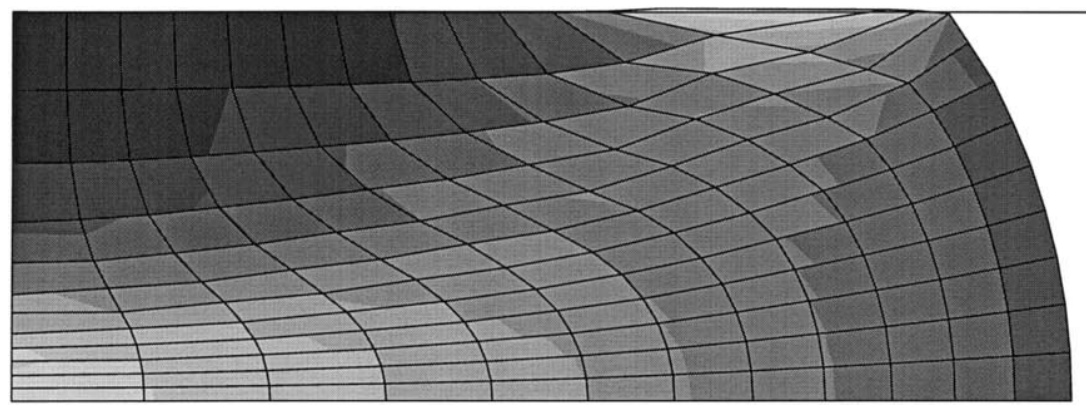

Fig. 6. Deformed shape at $60 \%$ upsetting with the plastic strain distribution (thermal softening not considered).

\section{Thermo-elastoplastic constitutive model}

A new thermo-elastoplastic model has been developed and implemented in the explicit dynamic code Stampack. The model employs the concept of hyperelasticity. The main advantage of hyperelastic models compared with hypoelastic formulations is the absence of need for calculation of derivatives satisfying the criteria of objectivity and of a need for integration of the constitutive equations $[8,9]$.

Formulation of the constitutive model for the thermo-elastoplastic material is an extension of that of the clastoplastic model present thermo-elastoplastic problems. I large thermo-elastoplastic deformations multiplicative decomposition (se mation gradient tensor $F$ into plastic parts, $F^{\mathrm{e}}, F^{\theta}$ and $F^{\mathrm{p}}$, respectively

Registe $F^{e} F^{\theta} F^{p}$ free at https//www.scipedia.c15in with

det $F^{\mathrm{p}}=1$,

$\boldsymbol{F}^{\theta}=J_{\theta} \boldsymbol{I}, \quad$ where $\quad J_{\theta}=\mathrm{e}^{3 \alpha\left(T-T_{0}\right)}$

The Almansi strain tensor $\boldsymbol{e}$ can be expressed by means of the deformation gradient tensor $\boldsymbol{F}$ as

$\boldsymbol{e}=\frac{1}{2}\left(\boldsymbol{I}-\boldsymbol{F}^{-\mathrm{T}} \boldsymbol{F}^{-1}\right)$

Analogically to Eq. (18) we define the elastic Almansi tensor $\boldsymbol{e}^{\mathrm{e}}$ (in the spatial configuration) and the thermal Almansi tensor tensor $\overline{\boldsymbol{e}}^{\theta}$ (in the intermediate configuration):

$\boldsymbol{e}^{e}=\frac{1}{2}\left(\boldsymbol{I}-\boldsymbol{F}^{\mathrm{e}-\mathrm{T}} \boldsymbol{F}^{\mathrm{e}-1}\right)$

$\overline{\boldsymbol{e}}^{\theta}=\frac{1}{2}\left(\boldsymbol{I}-\boldsymbol{F}^{\mathrm{e}-\mathrm{T}} \boldsymbol{F}^{\mathrm{e}-1}\right)$

After transforming the thermal Almansi tensor to the spatial configurations (the push-forward operation):

$\boldsymbol{e}^{\theta}=\frac{1}{2} \boldsymbol{F}^{\mathrm{e}-\mathrm{T}}\left(\boldsymbol{I}-\boldsymbol{F}^{\theta-\mathrm{T}} \boldsymbol{F}^{\theta-1}\right) \boldsymbol{F}^{\mathrm{e}-1}$

we can introduce the following additive relation:

$\boldsymbol{e}=\boldsymbol{e}^{\mathrm{e}}+\boldsymbol{e}^{\theta}+\boldsymbol{e}^{\mathrm{p}}$ defining the plastic Almansi tensor $\boldsymbol{e}^{\mathrm{p}}$. Applying the Lie derivative $L_{v}$ to all the components of the Almansi tensor in $\mathrm{Eq}$. (22) we obtain the additive decomposition of the deformation rate tensor

$d=d^{\mathrm{e}}+d^{\theta}+d^{\mathrm{p}}$

The stress response is characterized by means of the elastic free energy function of the form:

$\psi^{\mathrm{e}}=\frac{1}{2} \lambda \operatorname{tr}\left(e^{\mathrm{e}}\right)^{2}+\mu\left(e^{\mathrm{e}}: e^{\mathrm{e}}\right)$

where $\lambda$ and $\mu$ are the Lamé constants. With this form

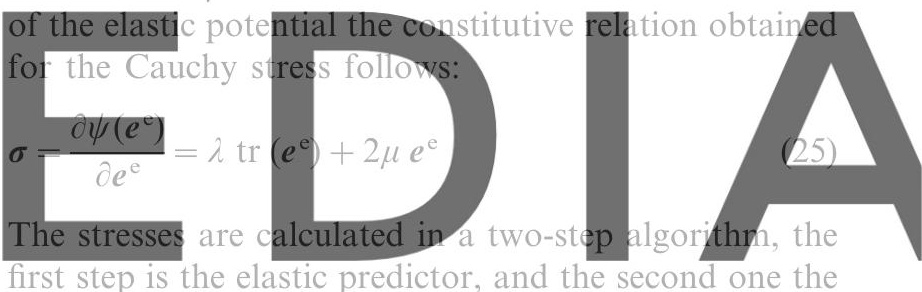

first step is the elastic predictor, and the second one the plastic corrector employing the radial return. For the

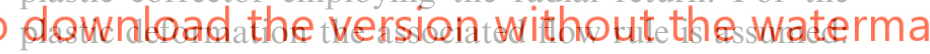

$d^{\mathrm{p}}=L_{v}\left(e^{\mathrm{p}}\right)=\dot{\lambda} \frac{\partial f}{\partial}$

with the Von Mises yield condition:

$f=\sqrt{\frac{\overline{3}}{2}}-\sigma_{\mathrm{Y}}\left(\bar{\varepsilon}^{\mathrm{p}}, T\right) \leq 0$

that accounts for the isotropic hardening and thermal softening:

$\sigma_{\mathrm{Y}}=\left[\sigma_{0}=\left(\sigma_{\infty}-\sigma_{0}\right)\left(1-e^{-\delta \bar{\varepsilon}^{\mathrm{p}}}\right)+H \bar{\varepsilon}^{\mathrm{p}}\right]\left[1-H_{\theta}\left(T-T_{0}\right)\right]$

where $\tau$ - the deviatoric Cauchy stresses, $\sigma_{0}$ and $\sigma_{\infty}$ initial and final yield stress, $\delta$-saturation constant, $H$-hardening modulus and $\mathrm{H}_{\theta}$ - thermal softening modulus.

The form of the elastic potential (Eq. (24)) is based on the assumption that the elastic part of the strains $\boldsymbol{e}^{\mathrm{e}}$ is small, which is fully justified for metals. It is assumed for reasons of simplicity and efficiency. Some authors starting from the same basic assumption expressed by Eq. (15), [11], have developed more general models, considering the possibility of large elastic deformations. Our formulation is simpler in implementation and no 
stamping problems. Computational plasticity, Proceedings of the Fifth International Conference, Barcelona, 1997, pp. 1781-1789.

[8] J.C. Simo, M. Ortiz, A unified approach to finite deformation elastoplastic anlaysis based on the use of hyperelastic constitutive relations, Comput. Meth. Appl. Mech. Eng. 49 (1985) $221-245$.

[9] C. García Garino, J. Oliver, Use of a large strain elastoplastic model for simulation of metal forming processes, in: J.L. Chenot, R. Wood, O.C. Zienkiewicz (Eds.), NUMIFORM '92, Balkema, 1992, pp. 467-472.

[10] C. García Garino, J. Oliver, A numerical model for elastoplastic large strain problems, in: D.R.J. Owen et al. (Eds.), Computational Plasticity, 1992, pp. 117-129.

[11] P. Wriggers, C. Miehe, M. Kleiber, J.C. Simo, On the coupled thermomechanical treatment of necking problems via finite element methods, Int. J. Num. Meth. Eng. 33 (1992) 869-883.

[12] C. García Garino, J. Rojek, E. Oñate, Simulation of sheet metal stamping processes using a solid finite strain model, in: L.A. Godoy, S.R. Idelsohn, P.A.A. Laura, D.T. Mook (Eds.), Applied Mechanics in the Americas, Proceedings of the Fourth Pan American Congress of Applied Mechanics, PACAM IV, Santa Fe, Argentina, vol. 1, 1995, pp. 97-102.

[13] NUMISHEET '96, Proceeding of the Third International Conference: Numerical Simulation of the 3-D Sheet Metal Forming Processes, Dearborn, MI, 1996.

[14] H. Lippmann, Metal Forming Plasticity, Springer, Berlin, 1979.

[15] ABAQUS, Examples manual, Version 5.6. Hibbit, Karlsson \& Sorensen, 1996.
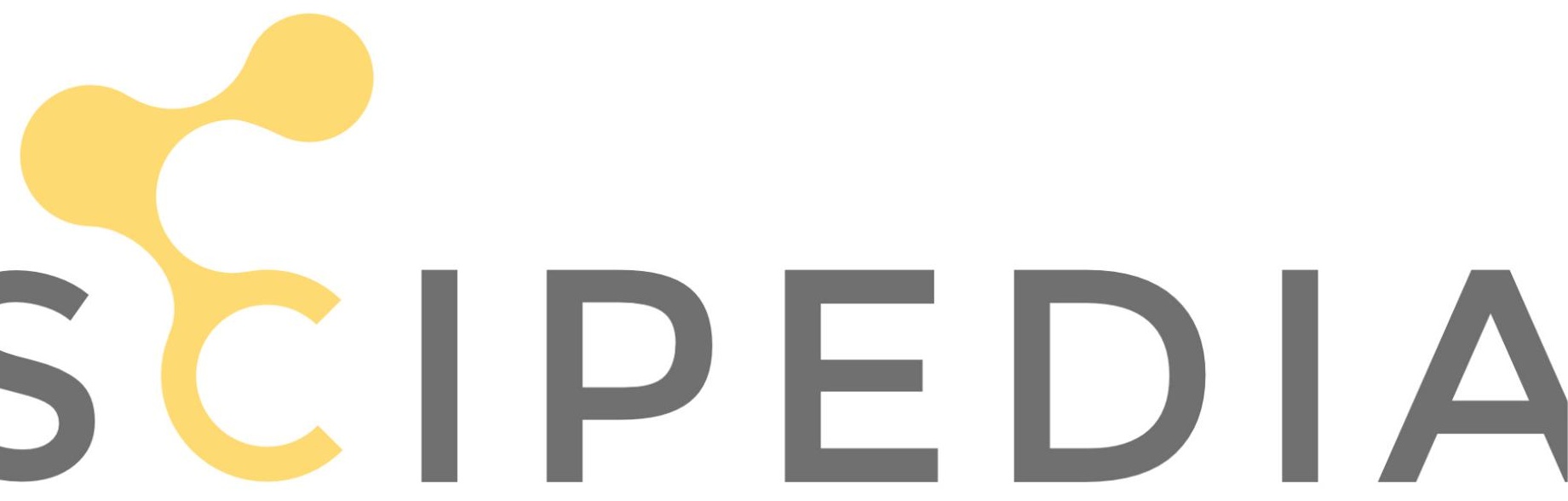